\title{
UCRL-TR-229205
}

LAWRENCE LIVERMORE NATIO NAL LABORATORY

Feasibility Study for Large Water-Based Neutron and Neutrino Detection

R.C.Svoboda, A.Bernstein, W.Coleman, S.A.Dazeley

March 19, 2007 
This document was prepared as an account of work sponsored by an agency of the United States government. Neither the United States government nor Lawrence Livermore National Security, LLC, nor any of their employees makes any warranty, expressed or implied, or assumes any legal liability or responsibility for the accuracy, completeness, or usefulness of any information, apparatus, product, or process disclosed, or represents that its use would not infringe privately owned rights. Reference herein to any specific commercial product, process, or service by trade name, trademark, manufacturer, or otherwise does not necessarily constitute or imply its endorsement, recommendation, or favoring by the United States government or Lawrence Livermore National Security, LLC. The views and opinions of authors expressed herein do not necessarily state or reflect those of the United States government or Lawrence Livermore National Security, LLC, and shall not be used for advertising or product endorsement purposes.

This work performed under the auspices of the U.S. Department of Energy by Lawrence Livermore National Laboratory under Contract DE-AC52-07NA27344. 


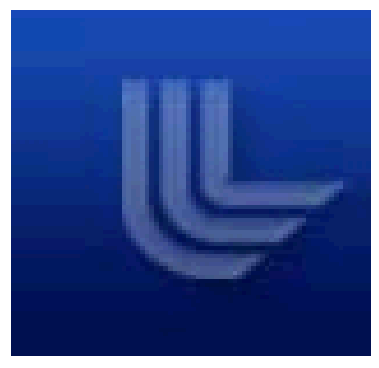

Lawrence Livermore National Laboratory

UCRL-XX-XXXXXX

\section{Feasibility Study for Large Water-Based Neutron and Neutrino Detection}

R.C.Svoboda, A.Bernstein, W.Coleman, S.A.Dazeley

22 February, 2007 
This document was prepared as an account of work sponsored by an agency of the United States Government. Neither the United States Government nor the University of California nor any of their employees, makes any warranty, express or implied, or assumes any legal liability or responsibility for the accuracy, completeness, or usefulness of any information, apparatus, product, or process disclosed, or represents that its use would not infringe privately owned rights. Reference herein to any commercial product, process, or service by trade name, trademark, manufacturer, or otherwise, does not necessarily constitute or imply its endorsement, recommendation, or favoring by the United States Government or the University of California. The views and opinions of authors expressed herein do not necessarily state or reflect those of him United States Government or the University of California, and shall not be used for advertising or product endorsement purposes.

This work was performed under the auspices of the U.S. Department of Energy (DOE) by the University of California, Lawrence Livermore National Laboratory (LLNL) under Contract W-7405-Eng-48. The project was funded by the Laboratory Directed Research and Development Program at LLNL. 


\begin{abstract}
The possibility of neutron and neutrino detection using water Cerenkov detectors doped with gadolinium holds the promise of constructing very large high-efficiency detectors with wide-ranging application in basic science and national security. This study addressed two major concerns about the feasibility of such detectors: (1) the transparency of the doped water to the ultraviolet Cerenkov light, and (2) the effect of the doped water on detector materials. We report on the construction of a 19-meter water transparency measuring instrument and associated materials test tank. The first sensitive measurement of the transparency of doped water at $337 \mathrm{~nm}$ has been made using this instrument $(>35$

meters). This transparency is sufficient to proceed to the next stage of building a prototype detector. Materials testing is not yet complete, as materials must be soaked for a year or more to assess the effects.
\end{abstract}

\title{
Introduction
}

Charged particles traveling through water with a speed greater than $\mathrm{c} / \mathrm{n}$, where $\mathrm{n}$ is the refractive index, will emit photons with a characteristic $1 / \lambda^{2}$ spectrum. These photons can be detected by placing light sensitive detectors, such as photomultiplier tubes, in the water. Since water is very transparent in the wavelength interval from about 300-500 nm, very large, inexpensive detectors can be realized. Such devices have been used for over twenty-five years in large mass neutrino experiments such as IMB, SNO, and SuperKamiokande.

Neutrons capturing on protons in the water emit a characteristic $2.2 \mathrm{MeV}$ gamma ray. Since water has a refractive index of 1.33, an electron must have a kinetic energy greater than $m_{e} c^{2}\left[n /\left(n^{2}-1\right)^{1 / 2}-1\right]=0.26 \mathrm{MeV}$ before it can emit light. Since the gamma energy is typically spread over several electrons in the absorption process, only a small amount of detectable light is emitted. For the Super-Kamiokande detector, simulations show that only a few phototubes would be hit - making detection essentially impossible against the background of roughly thirty hits expected from dark noise. Thus water Cerenkov detectors are not sensitive to neutron capture.

If one dopes the water with a nucleus that emits higher energy gammas, however, this situation can be improved. For example, gadolinium emits an $8 \mathrm{MeV}$ gamma cascade that simulations show would result in $\sim 35$ photomultiplier hits, which would be detectable with high efficiency. In addition, with a cross section of $\sim 40,000 \mathrm{~b}$ compared to $0.2 \mathrm{~b}$ for hydrogen, capture can be dominated by gadolinium even at small concentrations.

There are several potential problems in determining whether or not it is feasible to use gadolinium in water: (1) is the transparency still high enough to propagate Cerenkov photons long distances, (2) what will be the effects of the dopant on detector materials, and (3) is enough light generated by the $8 \mathrm{MeV}$ gamma cascade - as predicted by the computer simulations. This study addresses the first two questions. 


\section{Test Instrument Design and Construction}

In order to test water transparency and material compatibility of typical detector components, a test facility was designed and built that included a pure water system and mixing tank, a light transmission measurement arm, a waste storage tank, and a material exposure test tank (figure 1.)

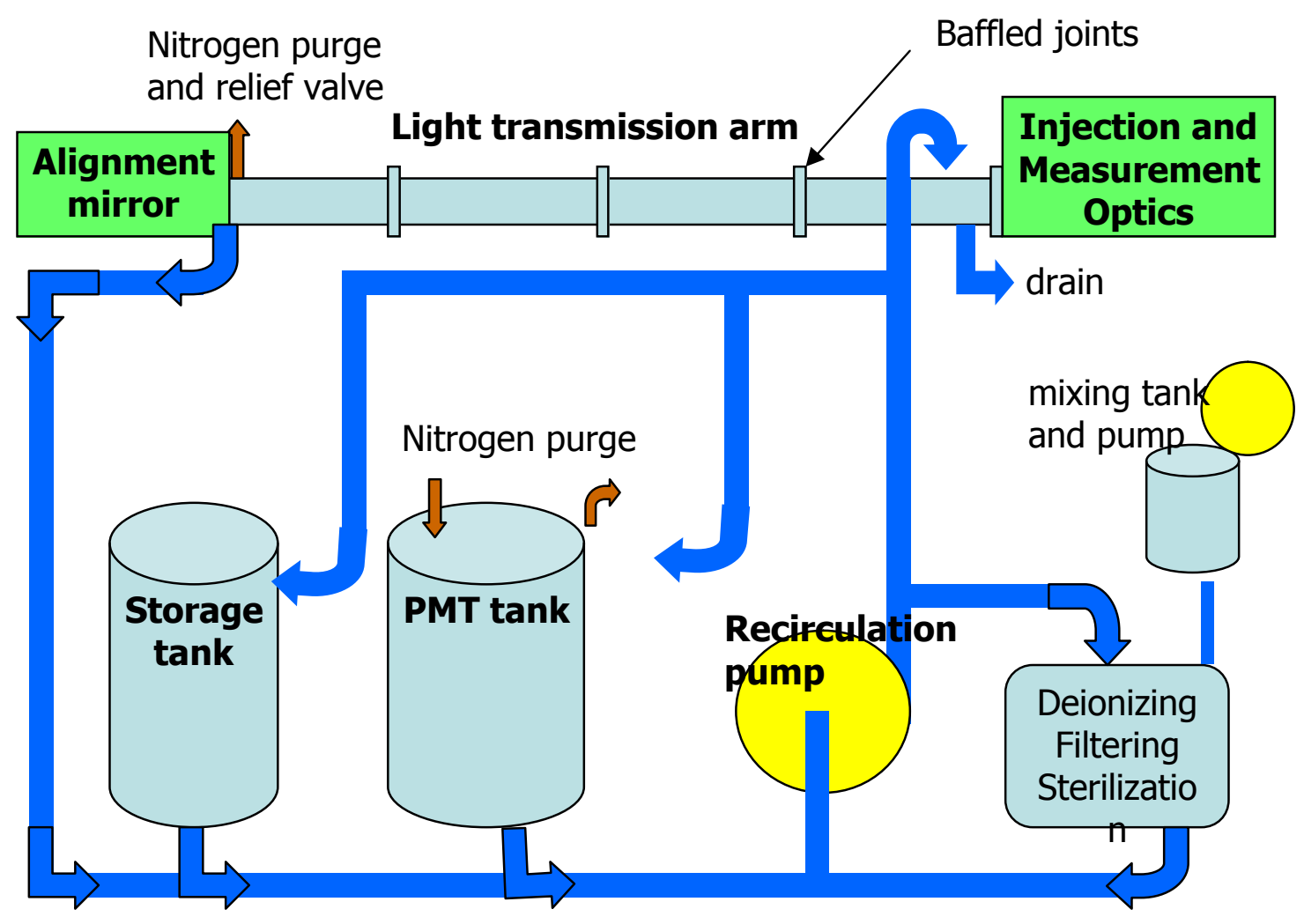

Figure 1: The test facility in Building 194. The system can produce 18 megaohm water from city water feed, and inject chemical dopants via a mixing tank. The water is also recirculated through 5 and 0.2 micron filters and an ultraviolet sterilizer to suppress bacterial growth. The water system services both the light transmission arm and the materials test tank, which holds two 50-cm diameter PMT's.

Since the transparency of pure water is typically 50-90 meters, a light transmission arm of length 9.54 meters is used. Since the light transverses the arm twice during testing (described below), the effective length is 20.08 meters. Thus a $10 \%$ change in the 
transparency of $90 \mathrm{~m}$ water would produce a $2.4 \%$ change in light intensity through the arm. This also sets the scale for the required instrument sensitivity and stability.

In order to keep the system chemically clean, only 304 stainless steel, acrylic, Teflon, viton, and polyethylene were used in construction. Components were cleaned with mild soap and water only (similar to typical large Cerenkov detectors) and rinsed with pure water before use. The system is designed to have a nitrogen blanket during operation in order to prevent oxygen mixing with the water - but for this first series of tests this was not ready - and thus the water is essentially oxygen saturated.

In addition to LLNL staff, university collaborators from Louisiana State University (W.Coleman) and U.C. Irvine (W.Kropp, H.Sobel, M.Smy, and M.Vagins) also assisted in the design and construction of the test facility. This project will be a major part of the Ph.D. thesis for Coleman, who spent roughly $40 \%$ of his time over the last year working with us at the lab on this project.

\section{The Water System}

The water system components were obtained commercially from South Coast Water and installed in a temporary hut erected outside Building 194 (figure 2). The outdoor location was necessary since the system contains noisy pumps and water chiller compressors that would make the noise levels inside the lab uncomfortable for researchers, and also cause unacceptable vibration of the sensitive optical system.

A water chiller is included in the system to allow cooling the water to $12-13 \mathrm{C}$ during the hot Livermore summers (not needed during the time period of this study). This further suppresses bacterial growth and also simulates more closely conditions in actual Cerenkov detectors. They are typically kept cool in order to reduce dark noise from the PMT's.

The system has a 0.2 and 5 micron filters to remove grit and dust. These filters are changed out at intervals of roughly 2-3 months. Included in the same loop are an ultraviolet sterilizer and conductivity monitors. With an appropriate valve lineup, the system can be set up for local (mixing tank only) recirculation, recirculation through the transmission arm (via pipes leading into the building), and/or recirculation to the materials test tank. Maximum recirculation rate is $2.5 \mathrm{gpm}$. Since the mixing tank + arm contain roughly $50+100=150$ gallons, turn over time is about one hour.

The system is fed via city water passed through portable demineralizer beds (not shown on the figure). Input water is typically has a resistivity of a few kohms. Using the approximate relation TDS $=(2 / 3)(1 \mathrm{M} \Omega / \rho)$ where $\rho$ is the resistivity in $\mathrm{M} \Omega$, the input water is roughly a few hundred ppm. Output of the demineralizer bed is typically $0.5 \mathrm{M} \Omega$, which corresponds to about 1-2 ppm. Note that water exposed to air will typically have a resistivity of about $2 \mathrm{M} \Omega$ due to the formation of carbolic acid, so this relationship is really only valid for degassed pure water, which saturates at about $18.6 \mathrm{M} \Omega$. 


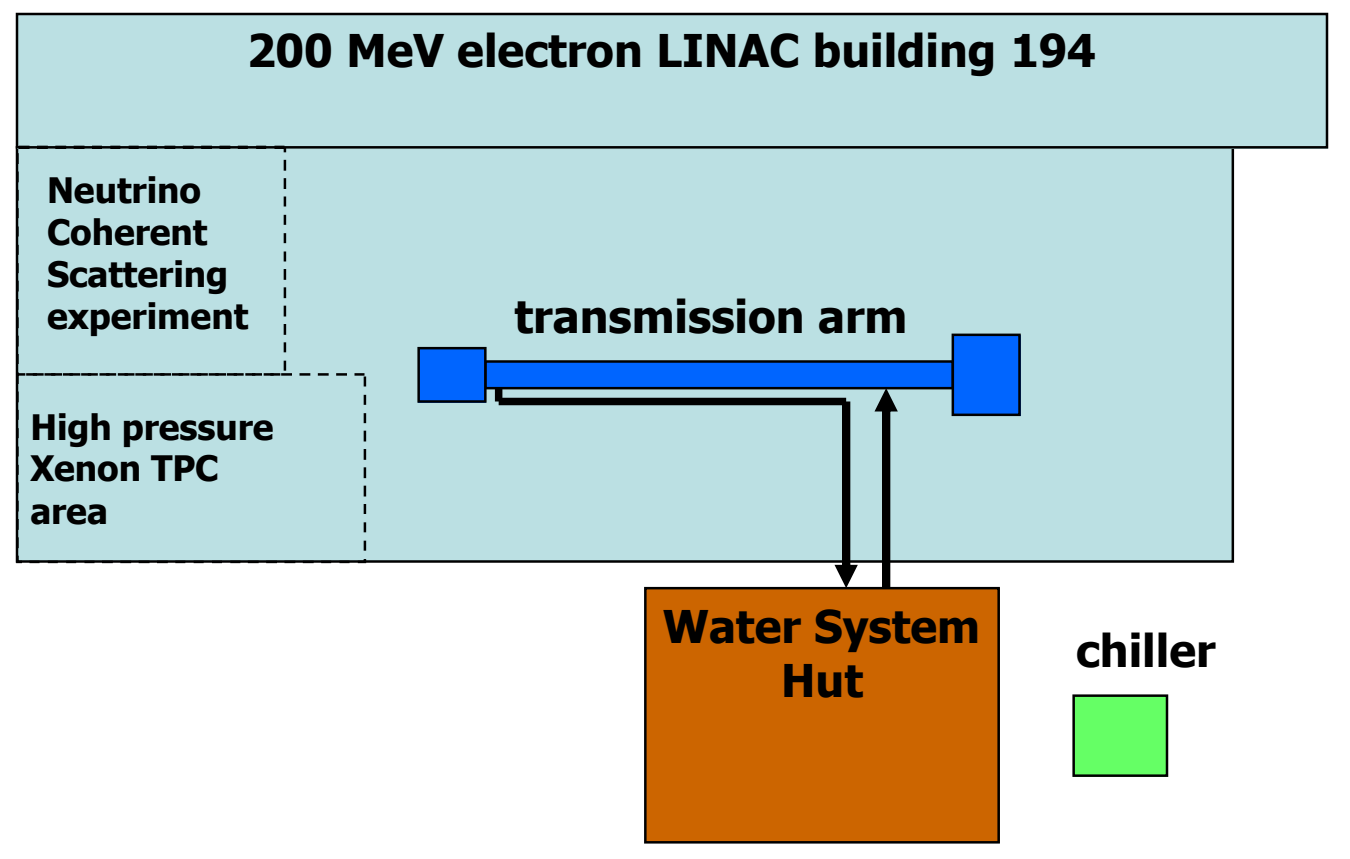

Figure 2: The water system hut is located outside B194 in order to prevent noise and vibration inside the lab. A water chiller keeps the water cool during the summer to avoid bacteria growth and simulate conditions inside typical Cerenkov detectors - which are kept as cool as possible to reduce dark noise.

From the portable demineralizer beds, the feed water passes into the mixing tanks, where it is then sterilized and filtered. A small deionizer then provides final stage purification up to over $18 \mathrm{M} \Omega$. Figure 3 shows the water system, mixing tank, portable resin beds, and materials test tank.

A 400 gallon storage tank (not shown) sits outside the water hut. This tank is used to store water that has gadolinium salt dissolved in it, and also provides a place to drain the entire system in an emergency. While the salt $\left(\mathrm{GdCl}_{3}\right)$ is not toxic, it is desirable to minimize all chemical discharge to the environment. Therefore the waste $(0.2 \%$ salt by weight) is stored for cleaning via demineralizer and appropriate disposal. The entire water hut (and transmission arm) is also lined with secondary containment in the event of a leak. 


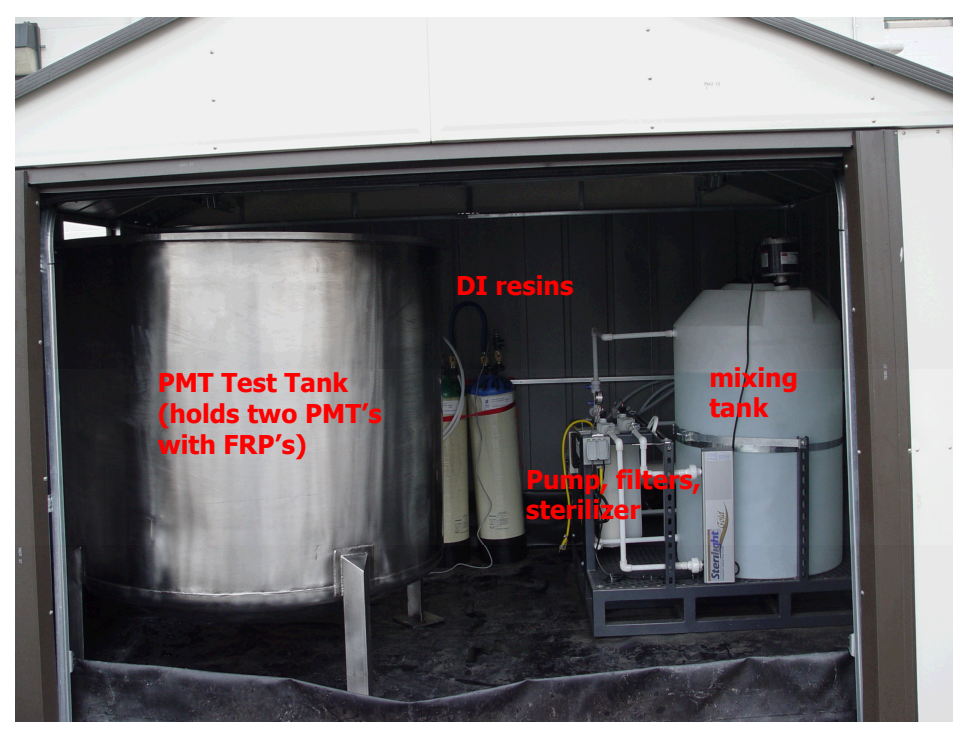

Figure 3: The water hut with the front sliding door in the open position. The purification system, test tank, and portable resin beds all sit inside a secondary containment berm.

\section{The Light Transmission Arm and Optics}

Figure 4 shows the Light Transmission Arm (LTA) and optical benches set up in the lab in B194. Light from a pulsed nitrogen dye laser is injected through an optical system (figure 5) located on a floating optical bench. In figure 5, the laser beam enters from the right side of the enclosure and passes through a beam splitter. Part of the beam is sent into a coated spherical integrator and part is sent through an acrylic window into the LTA. The integrated portion is collected via liquid light guide and piped into a white coated box viewed on one end by a mumetal shielded 2-inch PMT. This produced a "primary" (P) pulse that is recorded by waveform digitization electronics.

The other part of the split beam is sent through an acrylic window into the 9.54 meter long Light Transmission Arm (LTA). The window is covered by a black plastic slit $3 \mathrm{~cm}$ wide and $20 \mathrm{~cm}$ high to prevent late reflected light from entering. The LTA pipe itself is 8.5 inches $(22 \mathrm{~cm})$ in diameter, and contains four similarly-sized black slits spaced at equal distances along the interior. This removes scattered light from both the primary and reflected $(\mathrm{R})$ beam. At the far end, the $\mathrm{P}$ beam exits through an identical window and slit combination, reflects on a one-inch flat mirror, and re-enters the LTA as the R beam. Since incidence is very close to 90 degrees, very little light is reflected at the window/air interface.

The $\mathrm{R}$ beam returns down the LTA and exits roughly $10 \mathrm{~cm}$ below the incident beam and into another spherical coated integrator. This light is collected by another liquid light guide and piped into the PMT enclosure in a similar fashion as the P beam. Thus the same PMT records both the $\mathrm{P}$ and R pulse, cleanly separated in time by about $80 \mathrm{~ns}$. 


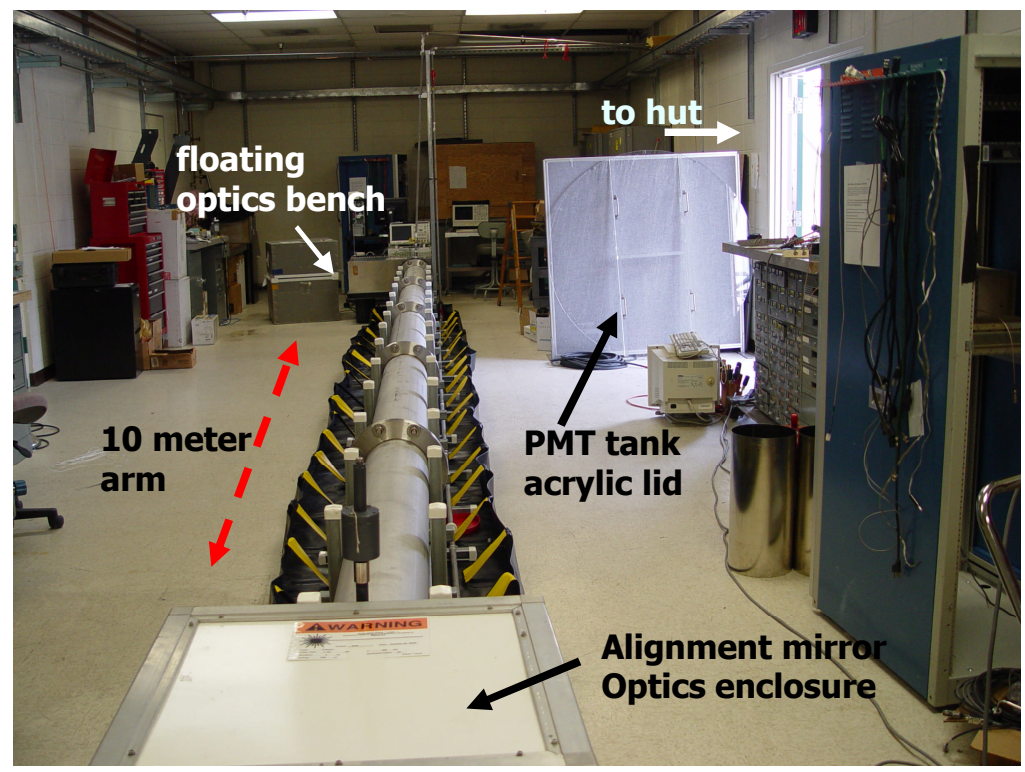

Figure 4: The LTA and associated optical benches. The laser beam is injected at the far end, traverses the arm, is reflected from a flat mirror in the mirror enclosure, and returns to the injection enclosure. The effective length is therefore $(2)(9.54)=20.08$ meters.

During normal operation, the $\mathrm{P}$ beam is never altered. All beam steering is done by adjusting the reflecting mirror in the far end enclosure, which is mounted on a three-axis optical stand for this purpose. In practice, it is necessary to realign the beam at periodic intervals by ensuring the $\mathrm{R}$ beam is exactly centered on the spherical integrator aperture. This can typically done in about 15 minutes, but it does introduce some instability in the system.

Although the exact reason for why the beam needs realignment is not certain, we think it may be due to thermal variations in the room causing very slight misalignment of the reflecting mirror, which is not on the same optical table as the other optics. Note that the LTA itself is floating on its own stand with a flexible cover connecting it to the optical table. Thus motions of the LTA do not really affect alignment.

It has also been noted that slight temperature and flow variations also cause beam misalignment. These variations can be caused when recirculation is changed through the LTA. Thus all data presented here is taken with recirculation turned off and room temperature stable. 


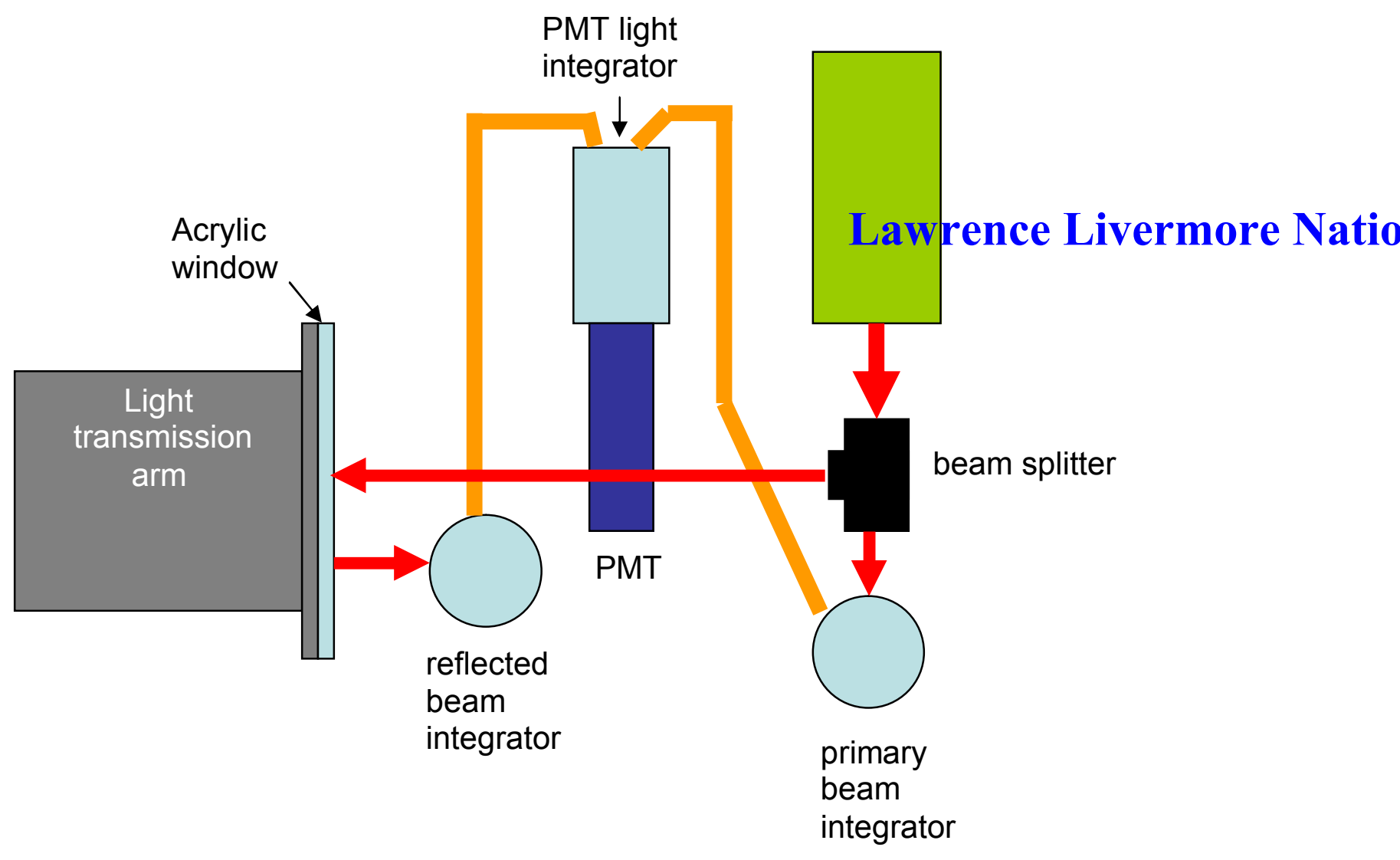

Figure 5: Schematic of injection and reflected beam optics. A laser beam (red arrows) passes through a splitter, where part is sent through the LTA and part is integrated and sent via liquid light guide (orange) to the PMT for measurement. The LTA component traverses the arm and is reflected into a second spherical integrator, where it is collected and sent to the PMT. Delay time between the primary and reflected is $80 \mathrm{~ns}$, which is sufficient to cleanly separate them in time.

\section{The Materials Test Tank}

The materials test tank was constructed at Royal Welding and Fabricating in Fullerton, CA. It is made from 304SS and is large enough to house two Super-Kamiokande 20-inch PMT's housed in their Fiber-Reinforced Plastic (FRP) anti-implosion housings. These PMT's are currently mounted in the tank, but testing awaits the availability of oxygenfree water, which has not yet been installed.

The top of the tank is covered by a large acrylic lid (figure 4) that is held in place to an oring seal by bolts. This lid is designed to have zero differential pressure between outside and inside, and to allow observation on the PMT's without opening the tank. 


\section{Results of Transparency Measurement}

Figure 6 shows a typical waveform digitizer output for a single laser shot at $337 \mathrm{~nm}$. The left pulse is the PMT response for the portion of the beam from the beam splitter that was integrated in the spherical integrator and piped to the PMT enclosure. The rightmost pulse is the same PMT response to the reflected beam that was integrated and piped to the PMT enclosure. The time separation of the two pulses is due to the extra distance traveled by the R pulse in water and air: $\Delta \mathrm{t}=(20.08)\left(1.33 / 3 \times 10^{8}\right)=89 \mathrm{~ns}$ for water plus $\sim 3 \mathrm{~ns}$ for air. The rise and decay time of the pulse is dominated by the response time of the integrators and is not representative of the laser pulse width, which is about $3 \mathrm{~ns}$. This particular waveform was taken in pure water.

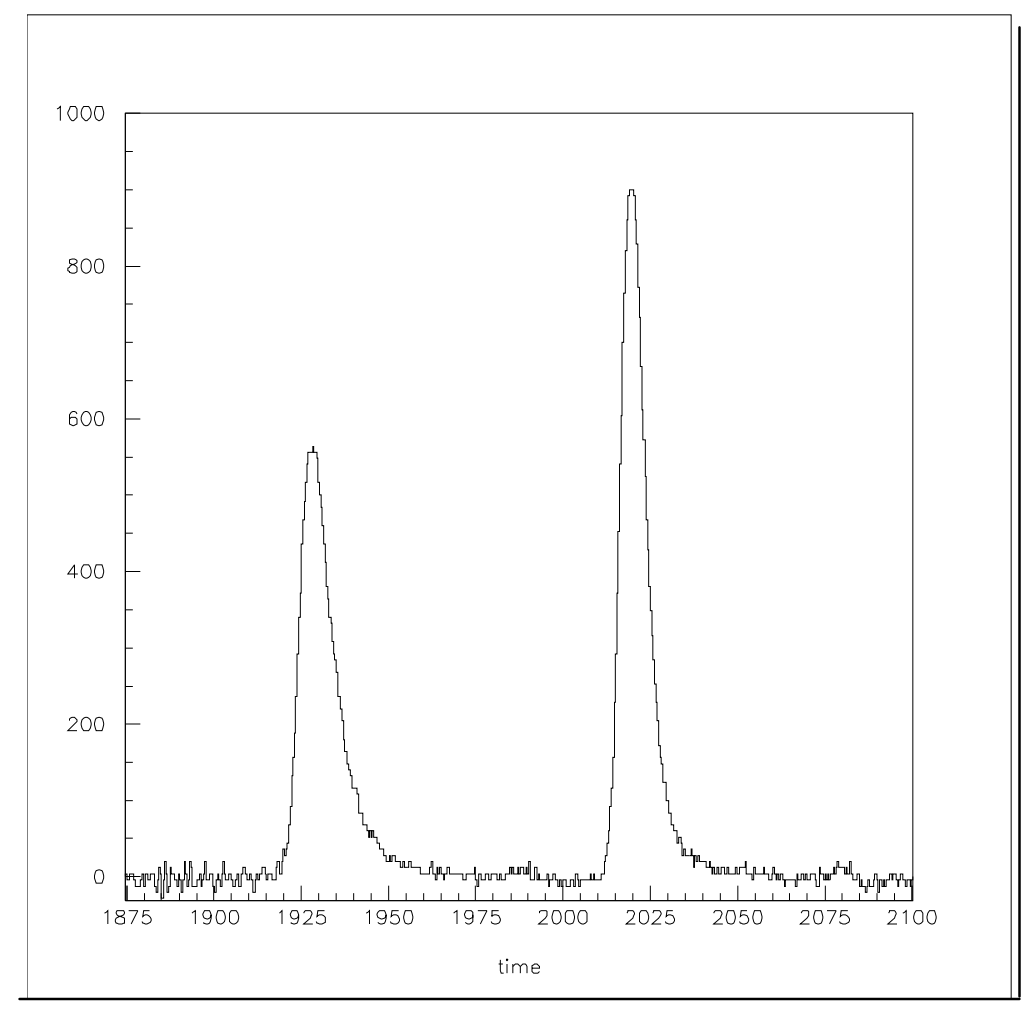

Figure 6: Waveform digitizer output of a single laser shot at $337 \mathrm{~nm}$. The left pulse is the output of the beam splitter and is proportional to the primary $(\mathrm{P})$ pulse. The right pulse is the reflected (R) pulse. The $\mathrm{x}$-axis time scale is in nanoseconds.

Figure 7 and figure 8 show the P ands R distribution of the pulse area for 100 laser shots. For each curve, a Gaussian has been fit to determine the mean and standard deviation. For both $\mathrm{P}$ and $\mathrm{R}$ the standard deviation is $3.4 \%$, and represents the pulse-to-pulse laser stability. The ratio of reflected to primary is a sensitive indicator of the relative water attenuation in the LTA. This can be understood as follows: 
Let the measured $\mathrm{P}$ pulse be proportional to the actual intensity $\left(\mathrm{I}_{0}\right)$ of the laser beam injected into the LTA:

$$
\mathrm{P}=\mathrm{a} \mathrm{I}_{0}
$$

Where a is the (unknown) constant of proportionality. Similarly for R and the intensity of the reflected pulse (I):

$$
\mathrm{R}=\mathrm{b} \mathrm{I}
$$

We know that:

$$
\mathrm{I}=\mathrm{I}_{0} \mathrm{e}^{-\alpha \mathrm{L}}
$$

where $\alpha$ is the attenuation coefficient and L is the length of the LTA times two. One can then define the ratio $(\rho)$ as:

$$
\rho=R / P=(b / a) e^{-\alpha L}
$$

Let $\rho_{1}$ be the ratio in pure water and $\rho_{2}$ be the ratio after adding $\mathrm{GdCl}_{3}$. Then it is easy to show that:

$$
\Delta \alpha=\alpha_{2}-\alpha_{1}=(1 / \mathrm{L}) \ln \left(\rho_{1} / \rho_{2}\right)
$$

Thus the unknown constants a and b cancel out, and we get the change in attenuation length with respect to pure water. The uncertainty in $\Delta \alpha$ will be given by (ignoring the uncertainty in L, which is very small):

$$
\sigma_{\Delta \alpha}=\left(\rho_{2} / \rho_{1}\right)(1 / L)\left\{\left(\sigma_{1} / \rho_{1}\right)^{2}+\left(\sigma_{2} / \rho_{2}\right)^{2}\right\}^{1 / 2}
$$

Data was collected in two different ways. The first way was to use the on-board averaging capabilities of the waveform digitizing system to give the average pulse area and standard deviation of 128 consecutive shots. This was very fast, taking only about one minute with the laser firing at $2 \mathrm{~Hz}$. Then $\rho$ was determined by taking the ratio of the means. The uncertainty in $\rho$ was in turn determined via standard techniques using the standard deviation about the mean for both pulses, respectively.

The problem with this method was that the $\mathrm{P}$ and $\mathrm{R}$ pulses are correlated: a slightly low intensity $\mathrm{P}$ means a slightly low intensity $\mathrm{R}$. The independent average method does not take advantage of this correlation. This can be remedied by taking the ratio $\mathrm{R} / \mathrm{P}$ on a pulse-to-pulse basis. 


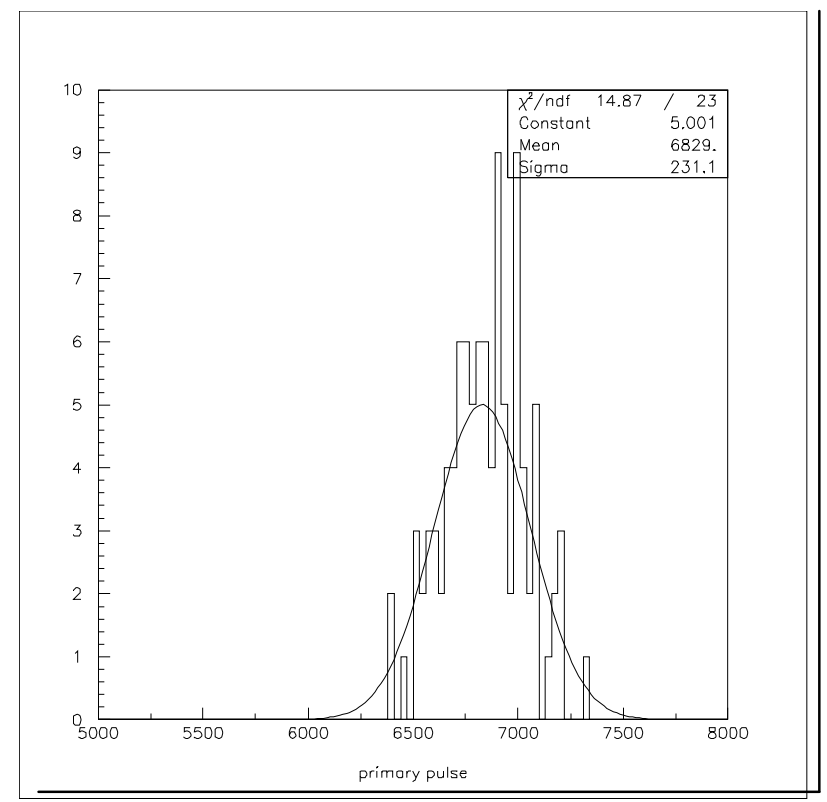

Figure 7: Histogram of the area of the $\mathrm{P}$ pulse for 100 laser shots at $337 \mathrm{~nm}$ in pure water. The variation is consistent with the pulse-to-pulse amplitude jitter expected for the laser.

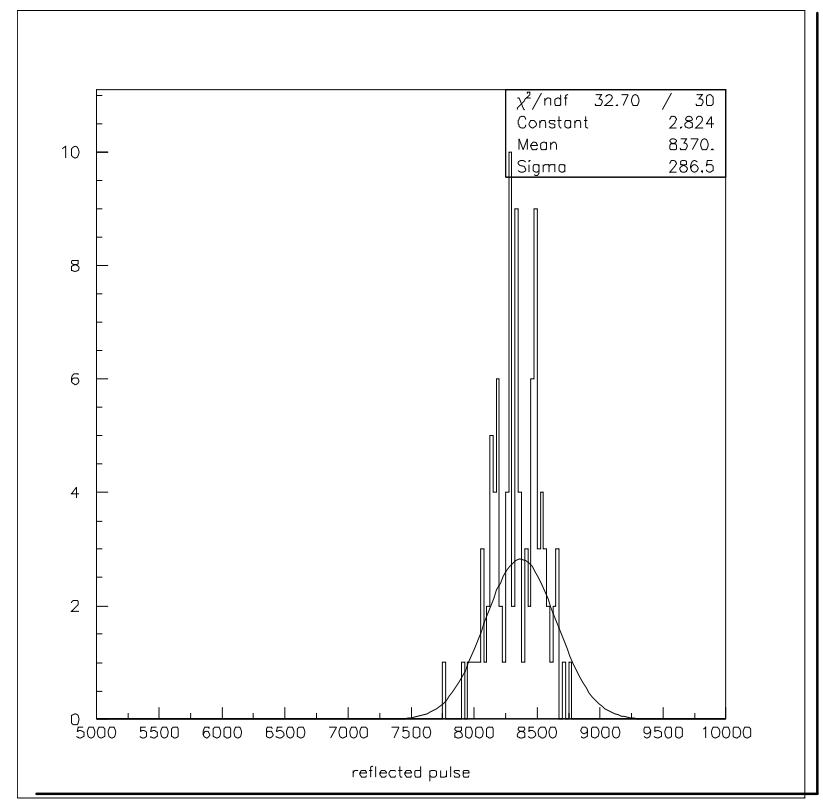

Figure 8: Same as figure 7, but for the R pulse.

Figure 9 shows the result of doing this. The variation in $\rho$ is only about $1 \%$ compared to $3.4 \%$ for the $\mathrm{R}$ and $\mathrm{P}$ pulses individually. This shows that it is possible to cancel out 
pulse-to-pulse laser variation. That being said, in practice it was found that $\rho$ would vary by $2-3 \%$ over the course of a few hours - much larger than the variation implied in a single 100 shot measurement. Thus we conservatively stick with the larger uncertainty estimate given in the equation above until this larger-than-expected variation can be understood.

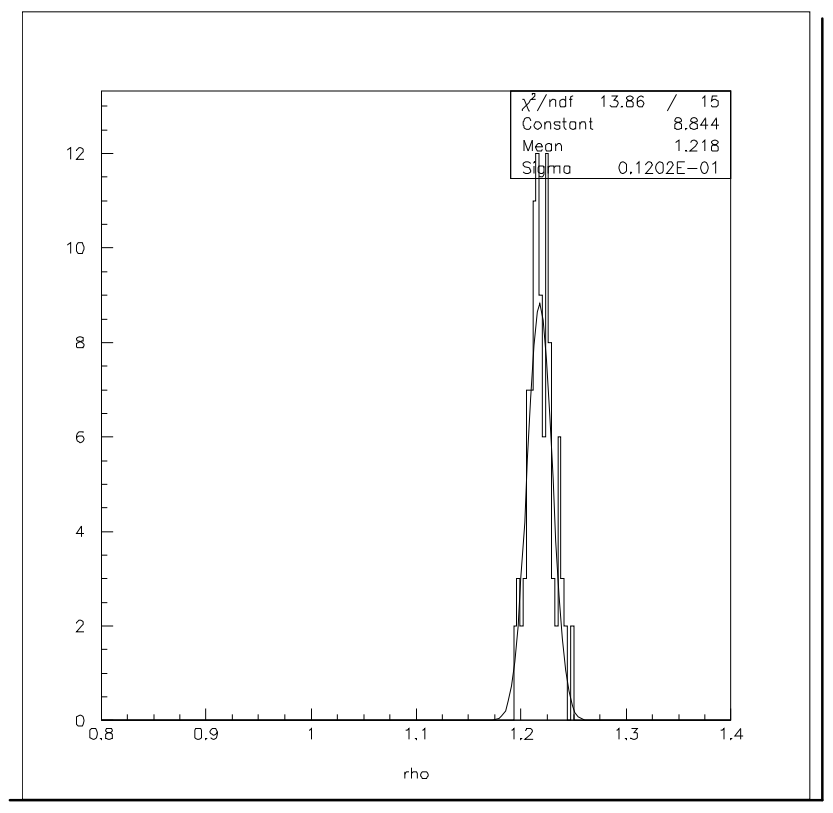

Figure 9: Histogram of the ratio R/P computed on a pulse-to-pulse basis using 100 laser shots at $337 \mathrm{~nm}$.

The system was run with pure water for about ten days prior to filling with $0.2 \% \mathrm{GdCl}_{3}$. The measured stability was on the order of $3 \%$ per day with no realignment, and about $1 \%$ per day with realignment. This was the case as long as only the reflecting flat mirror was aligned. If a primary beam alignment was done, the system was essentially "reset" and data runs could not be combined after this.

Figure 10 shows the measurement of $\rho$ in pure water from 12:00 12-Feb-07 to 20:00 12Feb-07. The error bars are those implied by uncorrelated P and R measurement, and are a conservative overestimate (as described above). The fitted value of $\rho$ is $1.2195(0.0171)$. Fitting to a line rather than a single value gives a slope much smaller than the uncertainty in the slope. Thus during this period $\rho$ did not change within the quoted uncertainty. Immediately after this run, a $\mathrm{GdCl}_{3} \cdot 6 \mathrm{H}_{2} \mathrm{O}$ solution was injected into the arm from the mixing tank, where it had been filtered and sterilized. $1.44 \mathrm{~kg}$ of the chemical was added - enough to make the solution $0.1 \%$ Gd by weight when thoroughly mixed.

During the mixing process, the reflected beam became unstable on a pulse-to-pulse basis due to the chemical mixing causing variations in the optical path. The arm was allowed to mix under active recirculation for twelve hours. At that point the reflected beam was again stable. Figure 11 shows the measurement of $\rho$ collected right after termination of 
the mixing recirculation. During these seven hours the system was again stable, with $\rho$ constant within the errors with a value of $1.0344(0.0201)$.

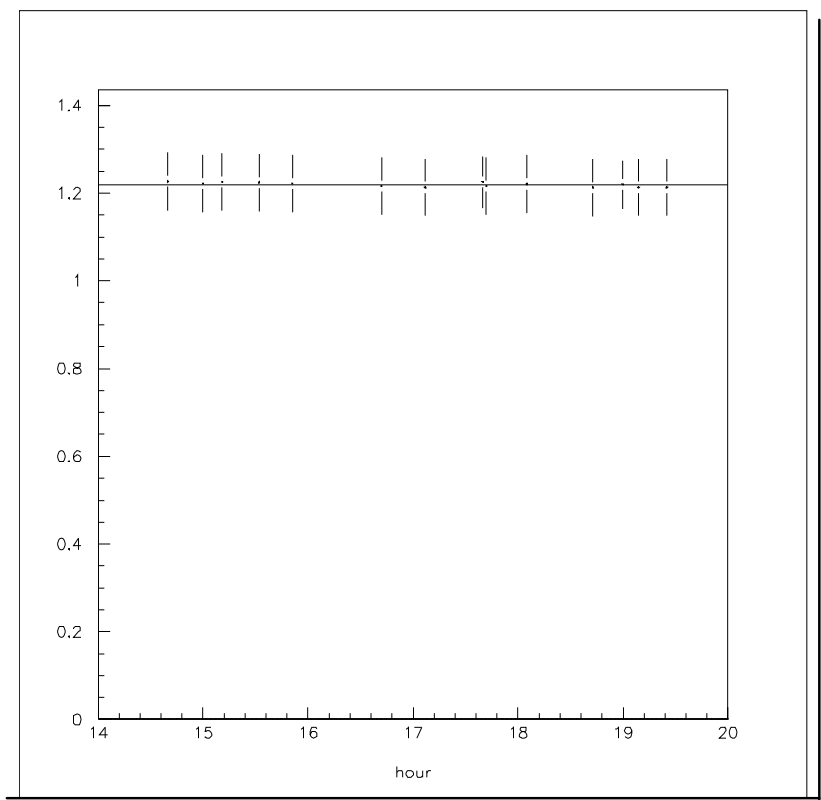

Figure 10: Measured values of $\rho$ over six hours with pure water in the LTA.

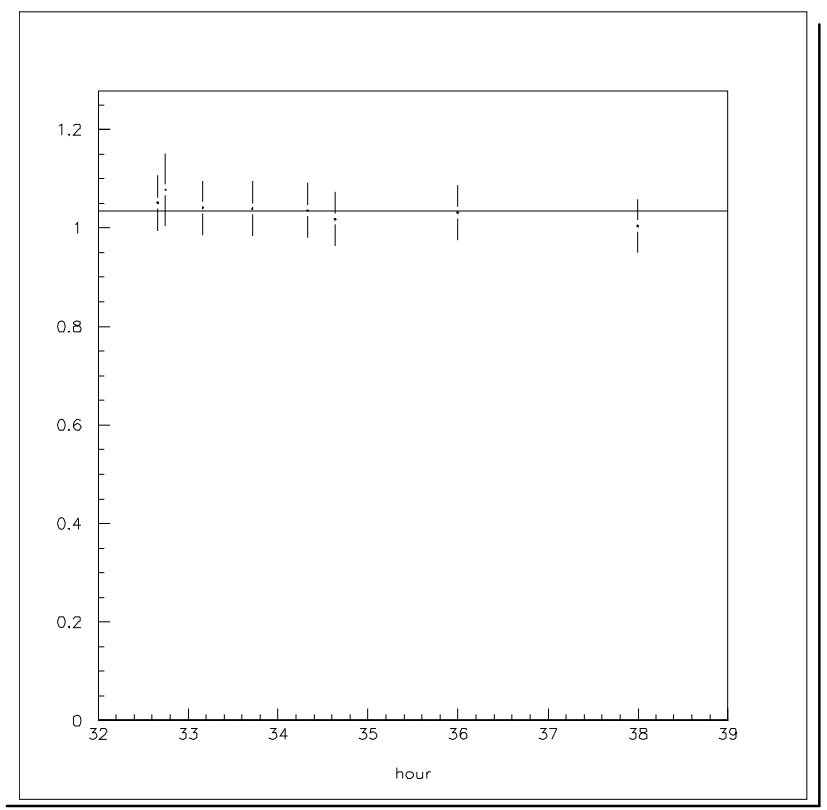

Figure 11: Measured values of $\rho$ over six hours in Gd-doped water. 


\section{Discussion}

Based on these two measurements, the attenuation coefficient changed by an amount $\Delta \alpha$ $=8.6(1.2) \times 10^{-3} \mathrm{~m}^{-1}$ between the fist run (pure water) and the second run $(0.1 \% \mathrm{Gd}$ by weight) . Measurements from Super-Kamiokande at $337 \mathrm{~nm}$ give the transparency of pure water at this wavelength of 53 meters $\left(\alpha=18.9 \times 10^{-3} \mathrm{~m}^{-1}\right)$. This implies a new attenuation length of $36(2)$ meters at $337 \mathrm{~nm}$, a decrease of roughly $30 \%$.

While such a decrease could be a problem for very large detectors such as SuperKamiokande or a megaton scale detector, it is not a problem for small detectors that have scale lengths less than 10 meters or so. In addition, $337 \mathrm{~nm}$ is at the edge of the sensitive region for Cerenkov detectors (roughly 300-500 nm). The behavior at other wavelengths is still to be determined.

It is also not clear if the decrease is due to absorption or scattering, and if it is the gadolinium chloride causing it directly or if it is being caused by chemical attack on the steel surface of the LTA. Visually, there was no rust visible when the pipe was opened for inspection, and the recirculation filters showed no more than "normal" discoloration similar to that of pure water. In addition, the system water was clear to the eye as it circulated through the white mixing tank.

\section{Publications and Proposals}

These preliminary results have shown that, at least for detectors on a size scale smaller than 4-5 meters, transmission of $\mathrm{UV}$ light in water doped with $\mathrm{GdCl}_{3}$ is sufficient to allow advancing to the next stage: namely to look at long-term stability and the transparency at longer wavelengths. In addition, before building large-scale practical detectors it is necessary to validate the computer simulations by actually building a prototype neutron detector. Testing of other materials (such as HDPE and PVC) in an independent test tank would also be desirable.

To this end, several follow-on proposals have been submitted building on this work:

(1) A FWP has been submitted to the DOE Office of Science to make an additional test tank and also a small-scale neutron detector. This proposal (also submitted to the National Science Foundation) is in conjunction with the RFP for R\&D associated with the Deep Underground Science and Engineering Laboratory (DUSEL). This type of detector would provide active neutron shields for sensitive experiments, and could also serve as an enhancement for a megaton scale neutrino detector.

(2) A proposal is being submitted to NA-22 to build a $2 \times 2 \times 2$ m neutron detector as a test bed for a passive cargo container scanning device. Such a device would have a very large acceptance and rely on coincident neutron detection to find SNM 
buried inside such containers. This would potentially be very fast, easy to operate, and environmentally friendly compared to existing detectors.

(3) The DOE Office of Science has provided funds at U.C. Davis for one of our members (Svoboda) who has a split appointment. These funds may be spent on equipment and students to help develop the LLNL test facility. Potential uses are for Dark Matter and neutrino experiments.

\section{Conclusions}

We have measured a $30 \%$ decrease in the attenuation length of $337 \mathrm{~nm}$ laser light after the addition of $\mathrm{GdCl}_{3}$ to pure water. The capability to measure at other wavelengths exists, and this will be done over the next few months by William Coleman, a student from LSU who will use this experiment as the topic for his Ph.D. thesis. This will provide crucial information needed to predict the behavior of gadolinium-doped water detectors vis-à-vis pure water ones. Final results will be also published in Nuclear Instrumentation and Methods (NIM) A after completion of his thesis.

Our preliminary conclusion (assuming that longer wavelengths are no worse than the 337 $\mathrm{nm}$ measurement) is that small detectors of length scales 10 meters or less will not suffer significant light loss due to gadolinium chloride doping. Long-term effects, however, are still to be measured. 